\title{
La Gran Simuladora: Tuberculosis Meníngea: Caso Clínico
}

\author{
The Great Simulator: Meningeal Tuberculosis: Clinical Case
}

\author{
Gabriela Alejandra López Robles*, Kristell Patricia Hawith Bautista
}

\section{RESUMEN}

La Tuberculosis (TB) es la enfermedad infecto-contagiosa más importante del mundo, asociada a alta morbimortalidad, principalmente es pulmonar, sin embargo puede afectar otros órganos y tejidos. La Organización Mundial de la Salud (OMS) 2015, estimó un millón de niños con TB en el mundo y 169,000 fallecidos. Según la OMS, Honduras ocupa el octavo lugar en carga de tuberculosis en el hemisferio occidental. Estos datos reflejan que continúa siendo un problema de salud pública prioritario. En países de alta endemicidad, la principal barrera es la pobreza y el escaso acceso al sistema sanitario. La incidencia de tuberculosis meníngea (TBM) se relaciona directamente con la prevalencia de TB pulmonar (TBP) y aunque solo representa alrededor del $1 \%$ de los casos de TB y 5\%-10\% de TB extrapulmonar, su presencia es preocupante por su elevada tasa de mortalidad (30\%) en aquellos pacientes que reciben tratamiento óptimo y de secuelas graves en el $50 \%$ de los sobrevivientes, por lo que el factor más importante que influye en el pronóstico es el diagnóstico precoz y el inicio temprano de tratamiento específico. El diagnóstico de la TBM con frecuencia representa un desafío para el médico; se necesita un alto grado de sospecha clínica para no retrasar el diagnóstico y tratamiento puesto que el cuadro clínico es inespecífico, heterogéneo y aunque el examen del líquido cefalorraquídeo (LCR) aporta datos sugestivos, habitualmente se inicia tratamiento empírico sin tener certeza de que el diagnóstico será confirmado. Las

\footnotetext{
* Residente de tercer año de Pediatría. EUCS, UNAH-VS

** Residente de tercer año de Pediatría.EUCS, UNAH-VS Dirigir correspondencia a: gabrielalopez@08hotmail.com Recibido: 17 de septiembre 2017 Aprobado: 20 de febrero 2018
}

manifestaciones clínicas y su duración son de vital importancia para su sospecha.

\section{PALABRAS CLAVES}

Tuberculosis, tuberculosis meníngea, tuberculosis cerebral.

\section{ABSTRACT}

Tuberculosis (TB) is the most important infectious-contagious disease in the world, associated with high morbidity and mortality, mainly pulmonary, but it can affect other organs and tissues. In the pediatric age it has been an occult epidemic because of its low infective capacity and lower incidence compared to adults. The World Health Organization (WHO) 2015 estimated one million children with TB in the world and 169,000 deaths. According to the WHO, Honduras ranks eighth in the burden of tuberculosis in the Western Hemisphere. These data reflect that it remains a priority public health problem. In countries of high endemicity, the main sweep is poverty and poor access to the health system. The incidence of meningeal tuberculosis (MTB) is directly related to the prevalence of pulmonary TB (TB) and although it represents only about $1 \%$ of cases of TB and $5 \%-10 \%$ of extrapulmonary TB, its presence is worrying because of its high Mortality rate $(30 \%)$ in those patients receiving optimal treatment and half of the survivors are left with severe sequelae, so the most important factor influencing the prognosis is early diagnosis and early initiation of specific treatment. The diagnosis of TBM often presents a challenge for the physician; A high degree of clinical suspicion is needed to avoid delaying diagnosis and treatment since the clinical picture is non-specific, heterogeneous and although cerebrospinal fluid 
(CSF) examination provides sugges- tive data, empirical treatment is usually initiated without being sure that the diagnosis Will be confirmed. The clinical manifestations and their duration are of vital importance for their suspicion.

\section{KEY WORDS}

Tuberculosis, tubercular meningitis, cerebral tuberculosis.

\section{INTRODUCCIÓN}

La TB es la enfermedad infecciosa más importante del mundo y una de las 10 primeras causas de muerte, aunque su incidencia y mortalidad están en franco retroceso. ${ }^{(1-2)}$

La TB cerebral es la forma más grave, constituye 5 a 15\% de los casos de TB extrapulmonar en los países en desarrollo y predomina en la población infantil. Se estima que se desarrolla aproximadamente en uno de cada 300 casos de TBP no tratada, acompañándose de afección miliar en $50 \%$. Es interesante que en 25 a $30 \%$ de los casos de TB cerebral no cursan con TBP. Se produce por diseminación hematógena de la TBP, en ella participan factores del huésped y de la bacteria que apenas se están caracterizando, así como la respuesta inmunológica local. ${ }^{(3)}$

Esta enfermedad produce cada año cerca de 1.6 millones de defunciones con nueve millones de nuevos casos y un tercio de la humanidad tiene infección latente, lo cual la convierte en la enfermedad infecto-contagiosa más relevante a nivel mundial.

Existen varias formas de TB cerebral, la más común es la meningitis tuberculosa, le siguen el tuberculoma, absceso tuberculoso, TB miliar cerebral, encefalopatía tuberculosa, encefalitis y arteritis tuberculosa.

La presentación clásica de la meningitis tuberculosa, es la de una infección subaguda, con pródromos inespecíficos como malestar general, pérdida de peso, fiebre de bajo grado y aparición gradual de cefalea, vómitos y confusión que conducen al coma y muerte si no se trata. En la exploración física puede encontrarse un grado variable de rigidez de cuello (suele estar ausente al inicio de la enfermedad), con la progresión de la enfermedad puede haber parálisis de nervios craneales VI, III, IV y VII, profundización del estado de coma, monoplejía, hemiplejía o paraplejiía en aproximadamente $20 \%$ de los casos. ${ }^{(4)}$

La meningitis tuberculosa afecta a todos los grupos de edad, pero es mucho más frecuente en población joven (especialmente niños) y personas infectadas por VIH (incrementa 5 veces más).

A pesar de que actualmente es curable, se necesita de cuatro antibióticos administrados por seis a nueve meses, lo que redunda en una alta tasa de abandono. Esta situación ha promovido recaídas y el surgimiento de cepas resistentes a varios antibióticos, lo cual complica aún más el tratamiento al incrementar su costo y toxicidad. La emergencia de cepas resistentes, la pandemia VIH/SIDA y el deterioro de los sistemas de salud pública en los países subdesarrollados han contribuido a empeorar la situación; de hecho, es en donde ocurren 95\% de los casos de TB activa y $98 \%$ de todas las defunciones. ${ }^{(2)}$

La vacuna actual BCG (Bacilo de Calmette Guerin) está lejos de ser la vacuna ideal, ya que el nivel de protección que confiere es muy irregular.

\section{CASO CLÍNICO}

Paciente masculino de 16 años de edad sin antecedentes patológicos de importancia, quien se presenta a emergencia acompañado de su madre con historia de presentar fiebre de 3 semanas de evolución, intermitente, subjetivamente alta, no cuantificada, 
sin predominio de horario, atenuada con acetaminofén 500 mg V.O. cada 6 hrs, además refiere que hace 3 semanas presentó cuadro de diarrea, líquida, fétida, sin moco, ni sangre, no pujo, ni tenesmo, aproximadamente 4-5 episodios diarios, por lo cual madre acudió a atención médica donde le realizaron estudios siendo el único positivo: Examen General de heces que reporto leucocitos de 21-40 por campo, eritrocitos 1-2 por campo, huevos de Hymenolepsis nana por lo que fue tratado en ese entonces con metronidazol 500 mg V.O. cada 12 hrs por 10 días. Debido a que la fiebre persistió y se agrega cefalea holocraneana, con intensidad de 5/10 en la escala del dolor, que se atenúa con acetaminofén, y los últimos 2 días previos presentando también vómitos de contenido alimentario de moderada cantidad, que le imposibilita la vía oral, hiporexia y tendencia al sueño lo llevan nuevamente a la emergencia.

Al recibirse nos encontramos a un paciente que lucía crónicamente enfermo, con cierto grado de desnutrición, pálido, con mucosas secas, hemodinámicamente estable, con tendencia al sueño, se decidió su ingreso para realizar estudios complementarios.

Se le realizaron los siguientes estudios: hemograma, PCR, prueba rápida de influenza, IgM para M. Pneumoniae, IgM para Herpes simple, Hemocultivos, BAAR, cultivo de secreción bronquial, PPD, resultando todos dentro de los límites normales, excepto IgM para M. Pneumoniae por lo que recibió tratamiento con Azitromicina durante 10 días. (Ver Figura No. 1).

Se realizó radiografía torácica donde se observa, imagen radiolúcida paracardíaca derecha, simulando una cavitación, versus neumonía. Por los resultados anteriores se solicitó TAC de Tórax (Ver Figura No. 2) la cual reporta: múltiples opacidades nodulares ramificadas en la periferia pulmonar de diámetro uniforme y distribución aleatoria intrapulmonar que sugiere cuadro de TB miliar; Además área de consolidación de 64 X 42 mm de tamaño, con broncograma aéreo y con presencia de cavernas en el interior localizado en el lóbulo superior del segmento posterior del pulmón derecho y cavernas en el lóbulo superior del pulmón izquierdo.

\section{Figura No. 1: Rayos X de Tórax}

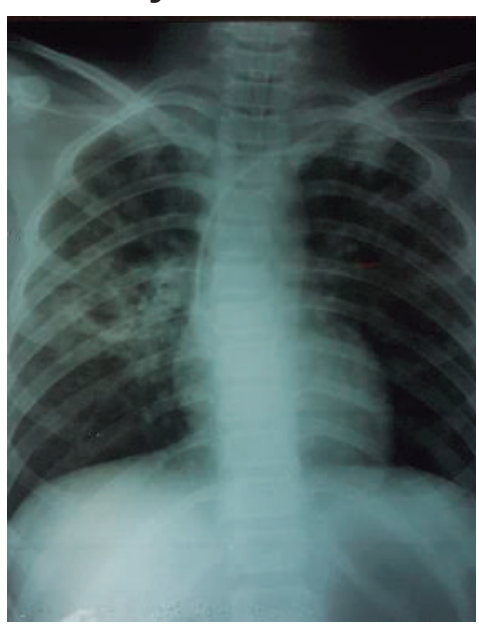

Fuente: Expediente Clínico

Figura No. 2: Tac de Tórax

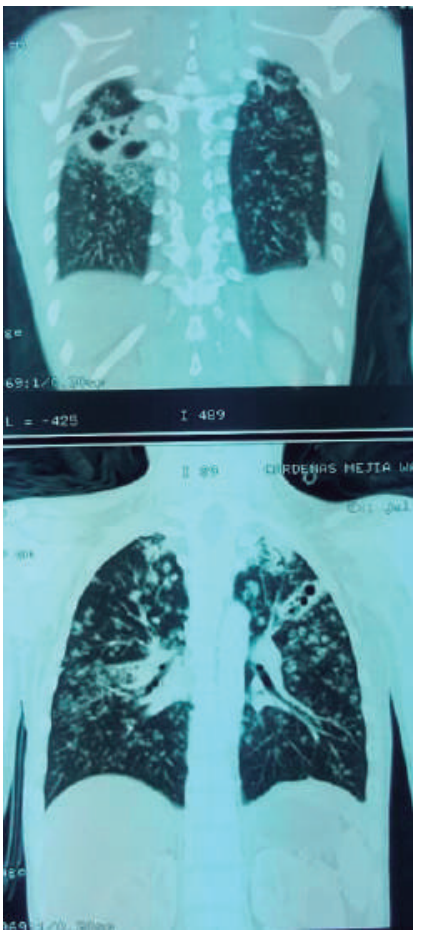

Fuente: Expediente Clínico 
Al examen físico lo llamativo fue su estado neurológico, con tendencia al sueño y ptosis palpebral derecha, pupilas isocóricas normoreaticas, babinsky presente en pie izquierdo y clonus, por lo que se le presentó al servicio de neurología, quien debido a su sintomatología neurológica que presentaba más la lesión sugestiva de cavitación a nivel pulmonar indicó que se debía descartar tuberculosis meníngea por lo que se indicó TAC cerebral y punción lumbar.

Tac cerebral reporta: presencia de lesión hipodensa a nivel paraventricular derecha. Se solicita IRM cerebral: se observan cambios hipointensos en T1 a nivel de fosa posterior, tienda del cerebelo y a nivel de la región temperoparietal derecho, y en T2 la señal cambia hiperintensa sobre todo en la base del cráneo y cerebelo. Con el medio de contraste se observa franco realce de las estructuras señaladas. Sistema ventricular conservado. Como conclusión: los cambios anteriormente mencionados están relacionados a un proceso inflamatorio agudo de la base del cráneo, sustancia blanca, bitemporales y biparietales de predominio derecho que en relación con la clínica la etiología es $\mathrm{M}$. Tuberculosis.

Citoquímica de LCR: proteínas: 169, glucosa: 21 , leucocitos 83 con linfocitos de $56.4 \%$ y neutrófilos de $43 \%$.

Debido a la sospecha diagnóstica de tuberculosis meníngea se inicia tratamiento antifímico con: isoniazida, rifampicina, etambutol y pirazinamida; y además se inicia prednisona. 36 horas posterior, presentó deterioro neurológico presentando Glasgow de 7 por lo que se traslada a Unidad de Cuidados Intensivos donde se realizó intubación orotraqueal permaneciendo intubado por 14 días; además inicio con bradicardia hasta $33 \mathrm{lpm}$ requirió 2 dosis de atropina al inicio normotenso pero después inicio con hipotensión por lo que requirió de administración de aminas vasoactivas: dobutamina y norepinefrina únicamente por 24 hrs.

Para terminar de confirmar dicho diagnóstico: Se envía PCR en LCR el cual reporta positivo.

Se envía genexpert de aspirado bronquial y reporta: presencia de $M$. tuberculosis y no hay resistencia a Rifampicina.

Actualmente paciente alimentándose por SNG, persiste su deterioro neurológico con Glasgow 10.

\section{DISCUSIÓN}

En la edad pediátrica la tuberculosis ha sido una epidemia oculta por su escasa capacidad infectiva y menor incidencia comparada con adultos; es la manifestación más devastadora de la TB y es causa importante de discapacidad neurológica en países de escasos recursos.

La TBM se presenta con más de 2 a 3 semanas de fiebre, rigidez de nuca y alteración del nivel de conciencia. La parálisis del nervio craneal y el papiledema se observan con mayor frecuencia en etapas avanzadas de la enfermedad.

Los pacientes también pueden presentar de manera tardía; hemiparesia, afasia, pérdida visual, convulsiones, movimientos coreiformes y complicaciones en el desarrollo. ${ }^{(5-7)}$

En niños mayores se presenta con síntomas inespecíficos como cefalea, vómitos y fiebre; similar a otras enfermedades pudiendo retrasarse el diagnóstico. La sospecha de TBM suele aparecer sólo algunos días o semanas después de la aparición de la enfermedad y no es diferente en los niños que han sido o no vacunados con Bacille CalmetteGürren. ${ }^{(8,9)}$ 
Siendo la fiebre, dolor de cabeza, anorexia y vómitos los que caracterizan el pródromo de la enfermedad en niños mayores como se presentó en nuestro caso, después de los primeros días de enfermedad, ocurren manifestaciones neurológicas relevantes, como parálisis de pares craneales. Mientras que el fracaso en el desarrollo, el escaso apetito, los vómitos y los trastornos del sueño son más comunes en los más jóvenes.

Etapa prodrómica: 7 días, la atrofia óptica en el examen de fondo, el déficit focal y los movimientos anormales fueron predictores independientes de TBM $(p<0,007)$ en un grupo de niños de 1 mes a 12 años.

En cuanto a las alteraciones en el LCR se encuentra: pleocitosis moderada con predominio de linfocitos, hiperproteinorraquia e hipoglucorraquia, datos que concuerdan con el estudio realizado del LCR en este caso clínico. ${ }^{(10,11)}$

La dificultad más importante se refiere al diagnóstico precoz, ya que en aquellos pacientes en los que se sospecha TBM con suficiente anticipación, el tratamiento actual es suficiente para lograr una cura completa en la mayoría de los casos, al menos cuando los patógenos son totalmente susceptibles y el tratamiento se cumple. ${ }^{(12)}$
Aproximadamente el $60 \%$ de los niños con TBM tiene evidencia radiológica de tuberculosis pulmonar, ${ }^{(12)}$ como en el caso del paciente estudiado que presentaba consolidación con broncograma aéreo y cavernas en el pulmón derecho.

En la TBM la enfermedad puede progresar incluso con tratamiento específico, puede desarrollar infarto, granulomas o hidrocefalia. La aparición de la respuesta paradójica en la TBM es una preocupación importante y representa un desafío para el médico. Un estudio publicado informa de que 11 de 33 pacientes se deterioraron dentro de las 6 semanas de tratamiento antituberculoso, mientras que en otro estudio, el $45 \%$ de los pacientes TBM desarrolló nuevo infarto cerebral durante el tratamiento. ${ }^{(13)}$

Los cortiocosteroides reducen la mortalidad disminuyendo la respuesta inflamatoria. ${ }^{(14)}$

Es importante que como pediatras podamos reconocer de forma temprana las manifestaciones clínicas de esta enfermedad para llegar a un diagnóstico precoz y tratamiento oportuno, evitando las complicaciones y disminuyendo la mortalidad. 


\section{REFERENCIAS BIBLIOGRÁFICAS}

1. Mellado Peña MJ, et al. Actualización del tratamiento de la tuberculosis en niños. An Pediatr (Barc). 2017. http://dx. doi.org/ 10.1016/j.anpedi.2017.05.013.

2. Hernández-Pando R, et al. Aspectos inmunopatológicos de la tuberculosis meníngea. Rev Invest Clin 2013; 65 (4): 349-356.

3. Kaviyil JE, Ravikumar R. Diagnosis of tuberculous meningitis: Current scenario from a Tertiary Neurocare Centre in India, Indian J Tuberc. (2017), http://dx. doi.org/ 10.1016/j.ijtb.2017.01.005.

4. Nguyen T.H. Maia and Guy E. Thwaites. Recent advances in the diagnosis and management of tuberculous meningitis. CNS infections. Febrero 2017. Vol 30. num.1.

5. H. Erdem et al. Diagnosis of tuberculous meningitis. Clin Microbiol Infect 2014; 20:0600-60810.1111/1469-0691.1247.

6. Guy E Thwaites, Ronald van Toorn, Johan Schoeman. Tuberculous meningitis: more questions, still too few answers. Lancet Neurol 2013; 12: 999-1010.

7. Jawad N, Jafri S, Naqvi S, et al. A Case Report on Complicated Tuberculous Meningitis. (May 04, 2017) Cureus 9(5): e1222. DOI 10.7759/cureus.1222.

8. Fonseca, et al. Extrapulmonary tuberculosis in Colombian children. The International Journal of Mycobacteriology. Volume 6. Issue 2. July 28, 2017, IP: 190.92.33.228].
9. Moliva et al. Inmune response to Bacillus Calmette-Guerin Vaccination. Frontiers in Immunology. April 2017 | Volume 8 | Article 407. doi: 10.3389/fim mu.2017. 00407.

10. Sharma S, et al. Cytokines do play a role in pathogenesis of tuberculous meningitis: A prospective study from a tertiary care center in India. Journal of the Neurological Sciences. doi: 10.1016/j.jns. 2017.06.001.

11. Patel VB, Theron G, Prestamistas $L$ y col Diagnostic Accuracy of Quantitative PCR (Xpert MTB/RIF) for Tuberculous Meningitis in a High Burden Setting: A Prospective Study. PLoS Med 2013;10 (10);e1001536.

12. Ronald vanToorn, FCP and Regan Solomons, MMED. Update on the Diagnosis and Management of Tuberculous Meningitis in Children. Semin Pediatr Neurol 21:12-18 2014 Elsevier.

13. Usha Kant Misra, Jayantee Kalita and Sanjeev Kumar Bhoi. Spectrum and outcome predictors of central nervous system infections in a neurological critical care unit in India: a retrospective review. Trans R Soc Trop Med Hyg 2014; 108 : 141-146. doi:10.1093/trstmh/tru 008.

14. Guy E Thwaites, Ronald van Toorn, Johan Schoeman. Tuberculous meningitis: more questions, still too few answers. The Lancet neurology. August 23, 2013 http://dx.doi.org/10.1016/S1474-4422 (13)70168-6 1. 